\title{
commentary Dissociative identity disorder needs re-examination
}

\author{
Peter Tyrer \\ COMMENTARY ON... DISSOCIATIVE IDENTITY DISORDER ${ }^{\dagger}$
}

Peter Tyrer is Emeritus Professor of Community Psychiatry in the Centre for Psychiatry at Imperial College,

London, and Honorary Professor in

Psychiatry at the University of

Nottingham, UK.

Correspondence Professor Peter

Tyrer, Imperial College,

7th Floor, Commonwealth Building,

Hammersmith Hospital, London W12

ONN, UK. Email: p.tyrer@imperial.ac.uk

First received 5 Apr 2019

Accepted 14 May 2019

\section{Copyright and usage}

(C) The Royal College of Psychiatrists 2019.

${ }^{\dagger}$ See this issue.

\section{SUMMARY}

Dissociative identity disorder (DID) is as real as any other psychiatric disorder but has been overdiagnosed by gullible clinicians, especially in forensic settings. Its classification has been poor, but the new ICD-11 classification, especially of partial DID, should help research and practice.

\section{DECLARATION OF INTEREST}

None.

\section{KEYWORDS}

Dissociative disorders; forensic mental health services; personality disorders.

Paris (2019) presents a strong argument against the casual use of the diagnosis of dissociative identity disorder (DID) in forensic settings, but goes much further in hoping that the concept of DID can be 'consigned to history'. This reminds me of Aubrey Lewis's magisterial rejoinder to Eliot Slater's follow-up studies of hysteria that suggested the diagnosis did not exist: 'A tough old word like hysteria dies very hard. It tends to outlive its obituarists' (Lewis 1975). Fifteen years ago Piper \& Merskey (2004) made similar predictions about DID, but it remains very much alive despite all the criticism.

Why is this? In responding it is fair to quote the over-used mantra, 'absence of evidence is not evidence of absence'. Paris is right to point out that there are no randomised trials of DID; virtually all the descriptions are case studies and there is sometimes a high level of gullibility in interpreting the evidence from these. This is particularly relevant in interpreting DID in forensic practice, but it would be wrong to suggest that practitioners in the area are unaware of the differences between simulation and truth (Brand 2016).

\section{Confusing literature}

There is a great deal of literature on DID, much of it poor, speculative and dominated by a few noisy product champions, and the recent review by
Dorahy et al (2014) describing it as 'a complex, valid and not uncommon disorder, associated with developmental and cultural variables, that is amenable to psychotherapeutic intervention' goes beyond the available data. But some facts are incontrovertible. DID is found not only in 'patients who have been coached' (Paris 2019), is often associated with early trauma and may last for many years in the absence of any obvious motivation explaining persistence.

\section{Trauma-dissociation is over-stated}

Where the research data have been overblown is in adopting the trauma-dissociation model as the only cause. Such cause has not been demonstrated; in my view, it could only be properly evaluated by a large cohort study starting in infancy and some are now planned (Huntjens 2019). Lynn et al (2014), after reviewing the current evidence, conclude that 'the field should now abandon the simple trauma-dissociation model and embrace multifactorial models that accommodate the diversity of causes'. Trauma alone will not do, and even though it dominates the literature on the subject it is freely acknowledged that this cannot be the only precipitant, as trauma lies behind a panoply of mental disorders (Temple 2019).

\section{DID in the courtroom: case law is not enough}

As for research on DID in forensic psychiatry, something must be done to improve the evidence base. There is a tendency for the discipline in general to accept a lower standard of evidence to merit acceptance (Tyrer 2015). It is not helped by evidence in criminal law being decided by single cases. In the rest of medicine evidence is decided by group studies; single cases are often outliers. In forensic work, however, to slightly misquote Tennyson, 'so careless of the group it seems, so careful of the single case'. And the sheer excitement and plausibility created by those who claim DID in court just adds to the drama in criminal cases and is exploited unmercifully in works of fiction. 


\section{A clinical case for partial DID}

DID is also described in the new ICD-11 classification. Apart from full dissociation of personality, in which there is no apparent knowledge of the other personality, a condition that is rare, there is also a description of partial dissociative identity disorder that many will find helpful. Again, this has limited verification, but in clinical experience is much more common. It is well described in the words of the classification:

'Partial dissociative identity disorder is characterized by disruption of identity in which there are two or more distinct personality states (dissociative identities) associated with marked discontinuities in the sense of self and agency. Each personality state includes its own pattern of experiencing, perceiving, conceiving, and relating to self, the body, and the environment. One personality state is dominant and normally functions in daily life, but is intruded upon by one or more non-dominant personality states (dissociative intrusions). These intrusions may be cognitive, affective, perceptual, motor, or behavioural. They are experienced as interfering with the functioning of the dominant personality state and are typically aversive. The non-dominant personality states do not recurrently take executive control of the individual's consciousness and functioning, but there may be occasional, limited and transient episodes in which a dis tinct personality state assumes executive control to engage in circumscribed behaviours, such as in response to extreme emotional states or during episodes of self-harm or the re-enactment of traumatic memories' (6B65 Partial dissociative identity disorder, World Health Organization 2019).

In my own clinical practice, partial DID makes a lot of sense. The 'non-dominant' personality can create havoc by temporarily taking over, particularly at times of stress. One of these 'non-dominants' I know, a truculent adolescent, managed to dispose of her husband's car keys when on a trip out, and the normal dominant personality spent many fruitless hours looking for them, completely spoiling the occasion. Is this partial DID or just plain unadulterated spite? I do not know. More research is needed, but in the end I predict that kernel of truth will remain.

\section{References}

Brand BL, Webermann AR, Frankel AS (2016) Assessment of complex dissociative disorder patients and simulated dissociation in forensic contexts. International Journal of Law and Psychiatry, 49(Pt B): 197-204.

Dorahy MJ, Brand BL, Sar V, et al (2014) Dissociative identity disorder: an empirical overview. Australian and New Zealand Journal of Psychiatry, 48: 402-17.

Huntjens RJC, Rijkeboer MM, Arntz A (2019) Schema therapy for Dissociative Identity Disorder (DID): rationale and study protocol. European Journal of Psychotraumatology, 10: 1571377.

Lewis A (1975) The survival of hysteria. Psychological Medicine, 5: 9-12.

Lynn SJ, Lilienfeld SO, Merckelbach H, et al (2014) The trauma model of dissociation: inconvenient truths and stubborn fictions. Psychological Bulletin, 140: 896-910.

Paris J (2019) Dissociative identity disorder: validity and use in the criminal justice system. BJPsych Advances, this issue.

Piper A, Merskey H (2004) The persistence of folly: critical examination of dissociative identity disorder. Part II. The defence and decline of multiple personality or dissociative identity disorder. Canadian Journal of Psychiatry, 49: 678-83.

Temple MJ (2019) Understanding, identifying and managing severe dissociative disorders in general psychiatric settings. BJPsych Advances, 25: $14-25$

Tyrer P, Duggan C, Cooper S, et al (2015) The lessons and legacy of the programme for dangerous and severe personality disorders. Personality and Mental Health, 9: 98-106.

World Health Organization (2019) ICD-11. WHO (https://icd.who.int). 